\title{
On the class of functions strongly starlike of order $\alpha$ with respect to a point
}

\author{
by ADAM LECKO (Rzeszów)
}

\begin{abstract}
We consider the class $\mathcal{Z}(k ; w), k \in[0,2], w \in \mathbb{C}$, of plane domains $\Omega$ called $k$-starlike with respect to the point $w$. An analytic characterization of regular and univalent functions $f$ such that $f(U)$ is in $\mathcal{Z}(k ; w)$, where $w \in f(U)$, is presented. In particular, for $k=0$ we obtain the well known analytic condition for a function $f$ to be starlike w.r.t. $w$, i.e. to be regular and univalent in $U$ and have $f(U)$ starlike w.r.t. $w \in f(U)$.
\end{abstract}

1. Introduction. Let $U_{r}=\{z \in \mathbb{C}:|z|<r\}, 0<r \leq 1$, denote the disk of radius $r$ in the complex plane $\mathbb{C}$ and $U=U_{1}$ denote the unit disk. We denote by $B(\xi, \varrho), \xi \in U, \varrho>0$, the hyperbolic open disk with hyperbolic center at $\xi$ and hyperbolic radius $\varrho$. We recall that

$$
B(\xi, \varrho)=\{z \in U: D(\xi, z)<\varrho\}=\left\{z \in U:\left|\frac{z-\xi}{1-\bar{\xi} z}\right|<R=\tanh \varrho\right\},
$$

where

$$
D(\xi, z)=\frac{1}{2} \log \frac{|1-\bar{\xi} z|+|z-\xi|}{|1-\bar{\xi} z|-|z-\xi|}=\operatorname{artanh}\left|\frac{z-\xi}{1-\bar{\xi} z}\right|
$$

denotes the hyperbolic distance on $U$ between $\xi$ and $z$.

For each $\alpha \in(0,1]$ we denote by $S^{*}(\alpha)$ the class of functions $f$ regular in $U$, normalized by $f(0)=f^{\prime}(0)-1=0$ and satisfying

$$
\left|\arg \left\{\frac{z f^{\prime}(z)}{f(z)}\right\}\right|<\alpha \frac{\pi}{2} \quad \text { for } z \in U,
$$

called strongly starlike of order $\alpha$. For each $\alpha \in(0,1]$ the class $S^{*}(\alpha)$ is a subset of the class $S^{*}=S^{*}(1)$ of starlike functions. Therefore each function in $S^{*}(\alpha)$ is univalent.

1991 Mathematics Subject Classification: Primary 30C45.

Key words and phrases: functions starlike with respect to a point, starlike functions, strongly starlike functions, $k$-starlike functions, geometric characterization. 
The classes $S^{*}(\alpha)$ were introduced by Brannan and Kirwan [1], and independently by Stankiewicz [4, 5] (see also [2, Vol. I, pp. 138-139]).

Brannan and Kirwan found a geometric condition called $\delta$-visibility which is sufficient for a function to be in $S^{*}(\alpha)$. Stankiewicz [5] obtained an external geometric characterization of strongly starlike functions. In [3] Ma and Minda presented an internal geometric characterization of functions in $S^{*}(\alpha)$ using the concept of $k$-starlike domains.

Using an idea similar to that in the paper of Ma and Minda we introduce the class $\mathcal{Z}(k ; w), k \in[0,2], w \in \mathbb{C}$, of domains $\Omega$ which will be called $k$-starlike with respect to $w \in \Omega$. For $w=0$ the class $\mathcal{Z}(k ; 0)$ consists of the $k$-starlike domains. For $k=0$ the class $\mathcal{Z}(0 ; w)$ consists of the domains $\Omega$ starlike w.r.t. $w$, which means that the line segment joining $w$ and an arbitrary point $\omega \in \Omega$ lies in $\Omega$.

We present an analytic characterization of the class $S^{\mathrm{g}}(k ; \xi, w)$ of functions $f$ which are regular and univalent in $U$ and have $f(U) \in \mathcal{Z}(k ; w)$, where $w=f(\xi)$ and $\xi \in U$. In other words, the internal geometric property of $k$-starlikeness w.r.t. an interior point is connected with the class of regular and univalent functions $f$ satisfying an analytic condition (3.1), which are called strongly starlike of order $\alpha$ w.r.t. $w$.

2. Domains and functions $k$-starlike w.r.t. a point. Let $k \in(0,2]$ be fixed. We denote by $K_{1}(k)$ and $K_{2}(k)$ two closed disks of radius $1 / k$ each centered at $1 / 2-i \sqrt{1 / k^{2}-1 / 4}$ and $1 / 2+i \sqrt{1 / k^{2}-1 / 4}$, respectively. For $k=0$ we set

$$
\begin{aligned}
& K_{1}(0)=\{v \in \mathbb{C}: \operatorname{Im} v<0\} \cup[0,1], \\
& K_{2}(0)=\{v \in \mathbb{C}: \operatorname{Im} v>0\} \cup[0,1] .
\end{aligned}
$$

For each $k \in[0,2]$ we define

$$
E_{k}=K_{1}(k) \cap K_{2}(k) .
$$

Of course, $E_{0}=[0,1]$. Each set $E_{k}, k \in(0,2]$, contains the points 0 and 1 on its boundary.

For $A, B \subset \mathbb{C}$ and $\omega \in \mathbb{C}$ we define

$$
\begin{aligned}
A B=\{u v \in \mathbb{C}: u \in A \wedge v \in B\}, & A \pm B=\{u \pm v \in \mathbb{C}: u \in A \wedge v \in B\}, \\
\omega A=\{\omega\} A, & \omega \pm A=\{\omega\} \pm A .
\end{aligned}
$$

For fixed $k \in[0,2]$ define

$$
\Gamma_{k}^{+}=\partial E_{k} \cap \partial K_{1}(k) \quad \text { and } \quad \Gamma_{k}^{-}=\partial E_{k} \cap \partial K_{2}(k) \text {. }
$$

Then $\Gamma_{k}^{+}$and $\Gamma_{k}^{-}$, for $k>0$, are closed circular arcs in the boundary of $E_{k}$ with endpoints 0 and 1 and with interiors lying in the upper and lower halfplane, respectively. Clearly, $\Gamma_{0}^{+}=\Gamma_{0}^{-}=[0,1]$. Throughout, $\Gamma_{k}^{+}$and $\Gamma_{k}^{-}$ will be treated as oriented arcs: from 1 to 0 and from 0 to 1 , respectively. 
For $k>0$ this means that the boundary of the set $E_{k}$ is positively oriented, i.e. in counterclockwise direction.

For $w, \omega \in \mathbb{C}$ let

$$
\begin{gathered}
\Gamma_{k}^{+}(w, \omega)=w+(\omega-w) \Gamma_{k}^{+}, \quad \Gamma_{k}^{-}(w, \omega)=w+(\omega-w) \Gamma_{k}^{-}, \\
E_{k}(w, \omega)=w+(\omega-w) E_{k} .
\end{gathered}
$$

Of course, $\Gamma_{k}^{+}=\Gamma_{k}^{+}(0,1), \Gamma_{k}^{-}=\Gamma_{k}^{-}(0,1)$ and $E_{k}=E_{k}(0,1)$.

For $w, \omega \in \mathbb{C}, w \neq \omega, \Gamma_{k}^{+}(w, \omega)$ will be oriented from $\omega$ to $w$, and $\Gamma_{k}^{-}(w, \omega)$ from $w$ to $\omega$. For $k>0$ this means that the boundary of $E_{k}(w, \omega)$ is positively oriented.

For every $z \in \Gamma_{k}^{+} \backslash\{0,1\}$ we denote by $\theta(z) \in[0, \pi / 2]$ the directed angle from $i z$ to the tangent vector to $\Gamma_{k}^{+}$at $z$. We also set $\theta(1)=\lim _{\Gamma_{k}^{+} \ni z \rightarrow 1} \theta(z)=$ $\arccos (k / 2)$ and $\theta(0)=\lim _{\Gamma_{k}^{+} \ni z \rightarrow 0} \theta(z)=\pi / 2$.

Similarly, for every $z \in \Gamma_{k}^{-} \backslash\{0,1\}$ we denote by $\vartheta(z) \in[-\pi / 2,0]$ the directed angle from $i z$ to the tangent vector to $\Gamma_{k}^{-}$at $z$ and we set $\vartheta(1)=$ $\lim _{\Gamma_{k}^{-} \ni z \rightarrow 1} \vartheta(z)=-\arccos (k / 2)$ and $\vartheta(0)=\lim _{\Gamma_{k}^{-} \ni z \rightarrow 0} \vartheta(z)=-\pi / 2$.

OBSERVATION 2.1. 1. If $z$ moves along $\Gamma_{k}^{+}, k \in(0,2]$, from 1 to 0 , then $\theta(z)$ strictly increases from $\theta(1)=\arccos (k / 2)$ to $\theta(0)=\pi / 2$. For all $z \in \Gamma_{0}^{+}, \theta(z)=\pi / 2$.

2. If $z$ moves along $\Gamma_{k}^{-}, k \in(0,2]$, from 0 to 1 , then $\vartheta(z)$ strictly increases from $\vartheta(0)=-\pi / 2$ to $\vartheta(1)=-\arccos (k / 2)$. For all $z \in \Gamma_{0}^{-}, \theta(z)=-\pi / 2$.

Definition 2.2. Fix $k \in[0,2]$. A domain $\Omega$ in the plane is called $k$ starlike with respect to the point $w \in \Omega$ provided that $E_{k}(w, \omega) \subset \Omega$ for every $\omega \in \Omega$.

The set of all $k$-starlike domains w.r.t. $w \in \mathbb{C}$ will be denoted by $\mathcal{Z}(k ; w)$. For simplicity of notation we denote the set $\mathcal{Z}(0 ; w)$ by $\mathcal{Z}(w)$ and the set $\mathcal{Z}(0 ; 0)$ of all domains starlike w.r.t. the origin by $\mathcal{Z}$.

REMARK 2.3. 1. 0 -starlikeness of $\Omega$ w.r.t. $w \in \Omega$ is exactly starlikeness w.r.t. $w$, i.e. the line segment joining $w$ and an arbitrary point $\omega \in \Omega$ lies in $\Omega$.

2. $k$-starlike domains w.r.t. the origin will be called $k$-starlike. These domains were considered in [3].

The following lemma is clear.

LEMma 2.4. If $0 \leq k_{1} \leq k_{2} \leq 2, w \in \mathbb{C}$ and $\Omega \in \mathcal{Z}\left(k_{2} ; w\right)$, then $\Omega \in \mathcal{Z}\left(k_{1} ; w\right)$.

Since $\mathcal{Z}(k ; w) \subset \mathcal{Z}(w)$ for all $k \in(0,2]$, every domain in $\mathcal{Z}(k ; w)$ is simply connected. 
Lemma 2.5. If $\Omega \in \mathcal{Z}(k ; w)$ for $k \in(0,2]$ and $w \in \Omega$, then $E_{k}(w, \omega) \backslash\{\omega\}$ $\subset \Omega$ for every $\omega \in \partial \Omega$.

Proof. Fix $\omega \in \partial \Omega$. By Lemma $2.4, \Omega$ is starlike w.r.t. $w$ so $[w, \omega) \subset \Omega$. Take the sequence $w_{n}=w+(1-1 / n)(\omega-w), n \geq 2$, in $[w, \omega)$. It is clear that $\lim _{n \rightarrow \infty} w_{n}=\omega$. Since $w_{n} \in \Omega$ it follows that $E_{k}\left(w, w_{n}\right) \subset \Omega$ for all $n \geq 2$. Therefore

$$
\bigcup_{n=2}^{\infty} E_{k}\left(w, w_{n}\right) \subset \Omega .
$$

Notice also that

$$
E_{k}\left(w, w_{n}\right) \subset E_{k}\left(w, w_{n+1}\right) \quad \text { for } n \geq 2 .
$$

Indeed, let $u \in E_{k}\left(w, w_{n}\right)$. Then there exists $\eta \in E_{k}$ such that $u=w+$ $\left(w_{n}-w\right) \eta=w+(1-1 / n)(\omega-w) \eta$. By starlikeness of $E_{k}$ we see that $\zeta=\left(1-1 / n^{2}\right) \eta \in E_{k}$. Consequently,

$$
\begin{aligned}
w+\left(w_{n+1}-w\right) \zeta & =w+\left(1-\frac{1}{n+1}\right)(\omega-w)\left(1-\frac{1}{n^{2}}\right) \eta \\
& =w+\left(1-\frac{1}{n}\right)(\omega-w) \eta=u,
\end{aligned}
$$

which means that $u \in E_{k}\left(w, w_{n+1}\right)$, so (2.2) is proved.

Now we prove that

$$
\text { Int } E_{k}(w, \omega) \subset \bigcup_{n=2}^{\infty} E_{k}\left(w, w_{n}\right) .
$$

To this end, let $u \in \operatorname{Int} E_{k}(w, \omega)$. Thus there exists $\eta \in \operatorname{Int} E_{k}$ such that $u=w+(\omega-w) \eta$. Let $a \in \partial E_{k}, a \neq 0$, be the point of intersection of $\partial E_{k}$ with the straight line joining the origin and $\eta$. It is clear that $\eta \neq a$ and therefore $\zeta=n \eta /(n-1) \in E_{k}$ for some $n \geq 2$. Hence

$$
\begin{aligned}
w+\left(w_{n}-w\right) \zeta & =w+\left(1-\frac{1}{n}\right)(\omega-w) \zeta=w+\frac{n-1}{n}(\omega-w) \frac{n}{n-1} \eta \\
& =w+(\omega-w) \eta=u .
\end{aligned}
$$

This means that $u \in E_{k}\left(w, w_{n}\right)$. Therefore (2.3) holds.

From (2.1) and (2.3) we obtain

$$
\text { Int } E_{k}(w, \omega) \subset \Omega \text {. }
$$

It remains to prove that if $v \in \partial E_{k}(w, \omega), v \neq \omega$, then $v \in \Omega$. Suppose, on the contrary, that there exists $v \in \Gamma_{k}^{+}(w, \omega), v \neq \omega$, such that $v \notin \Omega$. By (2.4) we can assume that $v \in \partial \Omega$.

Let $w_{0}$ be an arbitrary point lying on the open subarc of $\Gamma_{k}^{+}(w, \omega)$ joining $\omega$ and $v$, so $w_{0}=w+(\omega-w) \eta$ for some $\eta \in \Gamma_{k}^{+}$. The directed angle from the 
vector $i\left(w_{0}-w\right)$ to the tangent vector to $\Gamma_{k}^{+}(w, \omega)$ at $w_{0}$ is equal to $\theta(\eta)$. From Observation 2.1 and since $k>0$ it follows that $\theta(\eta)>\arccos (k / 2)$. But considering the set $E_{k}\left(w, w_{0}\right)$ we see that the directed angle from the vector $i\left(w_{0}-w\right)$ to the one-sided tangent vector to $\Gamma_{k}^{+}\left(w, w_{0}\right)$ at $w_{0}$ is equal to $\arccos (k / 2)$. Hence the open subarc of $\Gamma_{k}^{+}(w, \omega)$ joining $w$ and $w_{0}$ is contained in the interior of $E_{k}\left(w, w_{0}\right)$. Thus $v \in \operatorname{Int} E_{k}\left(w, w_{0}\right)$. If now $w_{0} \in \Omega$, then $E_{k}\left(w, w_{0}\right) \subset \Omega$, so $v \in \Omega$. If $w_{0} \in \partial \Omega$, then by (2.4) we have Int $E_{k}\left(w, w_{0}\right) \subset \Omega$, so $v \in \Omega$ also. Both cases contradict the assumption that $v \in \partial \Omega$.

If we assume that $v \in \Gamma_{k}^{-}, v \neq \omega$, and $v \notin \Omega$, then a similar analysis leads to a contradiction once again. This ends the proof of the lemma.

Let $\xi \in U$ and $w \in \mathbb{C}$. The set of all functions $f$ regular in $U$ such that $f(\xi)=w$ will be denoted by $\mathcal{A}(\xi, w)$.

Definition 2.6. Fix $k \in[0,2]$. A function $f \in \mathcal{A}(\xi, w)$, where $\xi \in U$ and $w \in \mathbb{C}$, univalent in $U$ will be called $k$-starlike w.r.t. $w$ if the domain $f(U)$ is $k$-starlike w.r.t. $w$, i.e. $f(U) \in \mathcal{Z}(k ; w)$.

The set of all functions $f \in \mathcal{A}(\xi, w), w=f(\xi)$, which are $k$-starlike w.r.t. $w$ will be denoted by $S^{\mathrm{g}}(k ; \xi, w)$.

We write $S^{\mathrm{g}}(\xi, w)$ for $S^{\mathrm{g}}(0 ; \xi, w)$. If $\xi=0$ and $w=f(\xi)=0$, then $k$-starlike functions w.r.t. the origin will be called $k$-starlike (see [3]). For $k=0, \xi=0$ and $w=f(\xi)=0$ we obtain the well known class $S^{\mathrm{g}}(0,0 ; 0)$ of starlike functions. This class will be denoted by $S^{\mathrm{g}}$.

Let us also introduce the following classes:

$$
S^{\mathrm{g}}(k ; w)=\bigcup_{\xi \in U} S^{\mathrm{g}}(k ; \xi, w), \quad S_{\xi}^{\mathrm{g}}(k)=\bigcup_{w \in \mathbb{C}} S^{\mathrm{g}}(k ; \xi, w) .
$$

The basic property of these classes is preservation of $k$-starlikeness w.r.t. $w$ on each hyperbolic disk centered at $\xi$, which can be formulated as follows:

TheOREM 2.7. A regular and univalent function $f$ is in $S^{\mathrm{g}}(k ; \xi, w)$, where $k \in[0,2], \xi \in U$ and $w \in \mathbb{C}$, if and only if for every $\varrho>0$ the domain $f(B(\xi, \varrho))$ is in $\mathcal{Z}(k ; w)$, where $w=f(\xi)$.

Proof. Suppose first that $f \in S^{\mathrm{g}}(k ; \xi, w)$, where $k \in[0,2], \xi \in U$ and $w=f(\xi)$. Hence $\Omega=f(U) \in \mathcal{Z}(k ; w)$. Fix $\varrho>0$ and set $\Omega(\xi, \varrho)=$ $f(B(\xi, \varrho))$. We will show that $E_{k}(w, \omega) \subset \Omega(\xi, \varrho)$ for all $\omega \in \Omega(\xi, \varrho)$.

Since $\Omega$ is $k$-starlike domain w.r.t. $w$, we see that $w+(\omega-w) v \in \Omega$ for all $\omega \in \Omega$ and $v \in E_{k}$. Thus the function

$$
g(z)=f^{-1}(w+(f(z)-w) v), \quad z \in U
$$

is well defined for each $v \in E_{k}$, regular in $U$ and $g(U) \subset U$. Since $g(\xi)=\xi$, Pick's Theorem, the invariant formulation of Schwarz's Lemma, shows that 
$g(B(\xi, \varrho)) \subset B(\xi, \varrho)$. Moreover, $g(B(\xi, \varrho))=B(\xi, \varrho)$ only if $g$ is a Möbius transformation which maps the unit circle into itself. From (2.5) we now get

$$
w+(\Omega(\xi, \varrho)-w) v=f(g(B(\xi, \varrho))) \subset \Omega(\xi, \varrho)
$$

for all $v \in E_{k}$. This implies that $w+(\Omega(\xi, \varrho)-w) E_{k} \subset \Omega(\xi, \varrho)$. Consequently, $w+(\omega-w) E_{k}=E_{k}(w, \omega) \subset \Omega(\xi, \varrho)$ for all $\omega \in \Omega(\xi, \varrho)$. This means that $\Omega(\xi, \varrho) \in \mathcal{Z}(k ; w)$.

Conversely, suppose that $f(B(\xi, \varrho))$ is in $\mathcal{Z}(k ; w)$, where $w=f(\xi)$, for every $\varrho>0$. Since

$$
f(U)=\bigcup_{\varrho>0} f(B(\xi, \varrho)),
$$

the assertion is immediate. This ends the proof of the theorem.

3. An analytic characterization of the class $S^{\mathrm{g}}(k ; \xi, w)$. In this section we present an analytic characterization of functions $f \in S^{\mathrm{g}}(k ; \xi, w)$.

The main theorem of this paper is the following.

Theorem 3.1. If $f \in S^{\mathrm{g}}(k ; \xi, w)$ for $k \in[0,2), \xi \in U$ and $w \in \mathbb{C}$, then

$$
\left|\arg \left\{\frac{(1-\bar{\xi} z)(z-\xi) f^{\prime}(z)}{f(z)-w}\right\}\right|<\alpha \frac{\pi}{2}, \quad z \in U,
$$

where $\alpha=(2 / \pi) \arccos (k / 2)$.

Conversely, let $\alpha \in(0,1], \xi \in U$ and $w \in \mathbb{C}$. If (3.1) is satisfied for a function $f$ regular in $U$, then $f \in S^{\mathrm{g}}(k ; \xi, w)$ for $k=2 \cos (\alpha \pi / 2)$.

Proof. For $f$ regular in $U$ and $\xi \in U$ we set $\Omega=f(U), \Omega(\xi, \varrho)=$ $f(B(\xi, \varrho))$ and $C(\varrho)=\partial B(\xi, \varrho)$ for $\varrho>0$.

1. We first consider the case $k=0$.

(i) Assume that $f \in S^{\mathrm{g}}(\xi, w)$, where $\xi \in U$ and $w \in \mathbb{C}$. Thus $w=f(\xi)$ and $\Omega \in \mathcal{Z}(w)$. By Theorem 2.7, also $\Omega(\xi, \varrho) \in \mathcal{Z}(w)$ for every $\varrho>0$. Therefore $\arg (f(z)-w)$ is well defined locally on the circle $C(\varrho)$. Let us parametrize $C(\varrho)$ as follows:

$$
C(\varrho): \quad z=z(t)=\frac{R e^{i t}+\xi}{1+\bar{\xi} R e^{i t}}, \quad t \in[0,2 \pi),
$$

where $R=\tanh \varrho \in(0,1)$. Hence we get

$$
\begin{aligned}
z^{\prime}(t) & =\frac{i\left(1-|\xi|^{2}\right) R e^{i t}}{\left(1+\bar{\xi} R e^{i t}\right)^{2}}=i \frac{1-|\xi|^{2}}{1+\bar{\xi} R e^{i t}} \cdot \frac{R e^{i t}}{1+\bar{\xi} R e^{i t}} \\
& =i\left(1-\bar{\xi} \frac{R e^{i t}+\xi}{1+\bar{\xi} R e^{i t}}\right) \frac{R e^{i t}-|\xi|^{2} R e^{i t}}{\left(1-|\xi|^{2}\right)\left(1+\bar{\xi} R e^{i t}\right)} \\
& =\frac{i(1-\bar{\xi} z)(z-\xi)}{1-|\xi|^{2}} .
\end{aligned}
$$


By starlikeness of $C(\varrho)$ w.r.t. $w$ it follows that the function

$$
[0,2 \pi) \ni t \rightarrow \arg (f(z(t))-w)
$$

is nondecreasing. Hence and by (3.3) we have

$$
\begin{aligned}
\frac{d}{d t} \arg (f(z(t))-w) & =\frac{d}{d t} \operatorname{Im} \log (f(z(t))-w) \\
& =\operatorname{Im}\left\{\frac{z^{\prime}(t) f^{\prime}(z(t))}{f(z(t))-w}\right\} \\
& =\frac{1}{1-|\xi|^{2}} \operatorname{Re}\left\{\frac{(1-\bar{\xi} z)(z-\xi) f^{\prime}(z)}{f(z)-w}\right\} \geq 0
\end{aligned}
$$

for all $z \in U \backslash\{\xi\}$. As $w=f(\xi)$ the function

$$
Q(z, \xi)=\frac{(1-\bar{\xi} z)(z-\xi) f^{\prime}(z)}{f(z)-w}, \quad z \in U \backslash\{\xi\},
$$

has a removable singularity at $z=\xi$ with

$$
Q(\xi, \xi)=\lim _{z \rightarrow \xi} \frac{(1-\bar{\xi} z)(z-\xi) f^{\prime}(z)}{f(z)-f(\xi)}=\frac{1}{1-|\xi|^{2}},
$$

where we used the fact that $f^{\prime}(\xi) \neq 0$ since $f$ is univalent in $U$. Hence the inequality (3.5) holds for $z=\xi$ also. Since $Q(\xi, \xi)>0$, the minimum principle for harmonic functions shows that for all $z \in U$ the inequality (3.5) is strict, i.e.

$$
\operatorname{Re} Q(z, \xi)>0 \quad \text { for } z \in U,
$$

which is equivalent to (3.1) for $\alpha=1$.

(ii) Conversely, let (3.1) be satisfied for $\alpha=1$ and fixed $f$ regular in $U$, i.e. (3.7) holds. From (3.7) we see that $Q$ has no pole and no zero in $U$. But this holds only when $w=f(\xi)$ and $f^{\prime}(z) \neq 0$ for all $z \in U$. In consequence, $f \in \mathcal{A}(\xi, w)$ and $f$ is locally univalent in $U$. Moreover, from (3.7) we have $f(z) \neq w=f(\xi)$ for all $z \in U \backslash\{\xi\}$. We conclude that the equation $f(z)-w$ $=0$ has a unique simple zero at $z=\xi$ on $U$. The argument principle now shows that

$$
\Delta_{C(\varrho)} \arg (f(z)-w)=\operatorname{Im}\left\{\int_{C(\varrho)} \frac{f^{\prime}(z)}{f(z)-w} d z\right\}=2 \pi
$$

for every $\varrho>0$. Hence applying once more the argument principle we deduce that the equation $f(z)-\omega=0$ has a unique solution for each $\omega \in \Omega(\xi, \varrho)$, which implies univalence of $f$ in $B(\xi, \varrho)$ for every $\varrho>0$. In consequence, $f$ is univalent in $U$.

Further, from (3.7) and (3.5) it follows that the function (3.4) is increasing so the curve $f(C(\varrho))$ and consequently the domain $\Omega(\xi, \varrho)$ are starlike 
w.r.t. $w$ for every $\varrho>0$. In this way, by Theorem 2.7 we see that $f(U)$ is starlike w.r.t. $w$, which means that $f \in S^{\mathrm{g}}(\xi, w)$.

2. (i) Let now $k \in(0,2)$ and $\alpha=(2 / \pi) \arccos (k / 2)$. Let $f \in S^{\mathrm{g}}(k ; \xi, w)$ with $w=f(\xi)$. Hence $\Omega=f(U) \in \mathcal{Z}(k ; w)$. We will prove that (3.1) holds, i.e.

$$
|\arg Q(z, \xi)|<\alpha \frac{\pi}{2} \quad \text { for } z \in U,
$$

for $\alpha=(2 / \pi) \arccos (k / 2)$.

For $z=\xi$ the inequality $(3.8)$ is clear since $Q(\xi, \xi)=1 /\left(1-|\xi|^{2}\right)$ is a positive real number.

Now we prove that (3.8) is true for all points on $C(\varrho)$ for every $\varrho>0$. Let $\gamma_{\varrho}$ denote the curve $\partial \Omega(\xi, \varrho)$ positively oriented. For each $z \in C(\varrho)$ we denote by $\tau(z)$ the tangent vector to $\gamma_{\varrho}$ at $\omega=f(z)$, i.e.

$$
\tau(z)=z^{\prime}(t) f^{\prime}(z(t)),
$$

where $z=z(t)$ is given by (3.2). From (3.3) we get

$$
\tau(z)=\frac{i(1-\bar{\xi} z)(z-\xi) f^{\prime}(z)}{1-|\xi|^{2}}, \quad z \in C(\varrho) .
$$

Let $\varphi(z), z \in C(\varrho)$, denote the directed angle from the vector $i(f(z)-w)$ to $\tau(z)$, i.e.

$$
\begin{aligned}
\varphi(z) & =\arg \{\tau(z)\}-\arg \{i(f(z)-w)\} \\
& =\arg \left\{\frac{i(1-\bar{\xi} z)(z-\xi) f^{\prime}(z)}{\left(1-|\xi|^{2}\right) i(f(z)-f(\xi))}\right\} \\
& =\arg \left\{\frac{(1-\bar{\xi} z)(z-\xi) f^{\prime}(z)}{f(z)-f(\xi)}\right\}=\arg Q(z, \xi) .
\end{aligned}
$$

Let $z \in C(\varrho)$ and $\omega=f(z)$. By Theorem 2.7 the domain $\Omega(\xi, \varrho)$ is in $\mathcal{Z}(k ; w)$. Therefore by a limit argument $E_{k}(w, \omega) \subset \overline{\Omega(\xi, \varrho)}$.

As was mentioned in Section 2, the boundary of the set $E_{k}(w, \omega)$ is positively oriented. Let $s_{1}$ and $s_{2}$ be one-sided tangent vectors to the arcs $\Gamma_{k}^{+}(w, \omega)$ and $\Gamma_{k}^{-}(w, \omega)$ at $\omega$, respectively, and let $p_{1}$ and $p_{2}$ be the halflines starting from $\omega$ with directional vectors $s_{1}$ and $s_{2}$, respectively. We denote by $V$ the closed sector bounded by $p_{1}$ and $p_{2}$ with vertex $\omega$ for which Int $V \cap \operatorname{Int} E_{k}(w, \omega)=\emptyset$. The normal line to the vector joining $w$ and $\omega$ and going through $\omega$ divides the plane into two closed half-planes, one of them containing $E_{k}(w, \omega)$. Consequently, one of the two closed half-lines starting from $\omega$ and normal to the vector joining $w$ and $\omega$ lies in $V$; denote it by $p$. Then $p$ divides $V$ into two closed sectors with vertex at $\omega$ : $V_{1}$ bounded by $p_{1}$ and $p$, and $V_{2}$ bounded by $p_{2}$ and $p$. Since $E_{k}(w, \omega)$ is symmetric w.r.t. the straight line going through $w$ and $\omega$ which is normal to $p$, we see that $p$ 
bisects $V$. From the assumption that $\Omega(\xi, \varrho)$ is $k$-starlike w.r.t. $w$ it follows that the tangent line to $\gamma_{\varrho}$ at $\omega$ cannot intersect the interior of $E_{k}(w, \omega)$. Therefore the tangent vector $\tau(z)$ lies in $V$.

If $\tau(z)$ lies in $V_{1}$, then $\varphi(z)$ is nonnegative and in view of (3.9) we obtain

$$
\varphi(z) \leq \arg \left\{s_{1}\right\}-\arg \{i(f(z)-f(\xi))\}=\theta(1)=\arccos \frac{k}{2}=\alpha \frac{\pi}{2} .
$$

If $\tau(z)$ lies in $V_{2}$, then $\varphi(z)$ is nonpositive and using again (3.9) we have

$$
\varphi(z) \geq \arg \left\{s_{2}\right\}-\arg \{i(f(z)-f(\xi))\}=\vartheta(1)=-\arccos \frac{k}{2}=-\alpha \frac{\pi}{2} .
$$

In consequence, the inequalities (3.10) and (3.11) are true for every point in $C(\varrho)$. As $\varrho$ was arbitrary, they are satisfied in $U$.

Suppose that equality holds in (3.10). Then by the maximum principle for harmonic functions it holds for every point in $U$. But this is impossible since $Q(\xi, \xi)$ is a real number. Therefore the inequality (3.10) is strict, and similarly for (3.11).

(ii) Conversely, let $\alpha \in(0,1)$ and assume that (3.1) is satisfied for $f$ regular in $U$, i.e. (3.8) holds. As in Part 1(ii) we can prove that $w=f(\xi)$ and therefore $f \in \mathcal{A}(\xi, w)$.

The inequality (3.8) is clearly true for $\alpha=1$ also. But, as was shown in Part 1(ii), this implies that $f \in S^{\mathrm{g}}(\xi, w)$ and therefore $f$ is univalent in $U$. Thus we need to prove that $f(U) \in \mathcal{Z}(k ; w)$ for $k=2 \cos (\alpha \pi / 2)$.

Suppose, on the contrary, that $f(U)$ is not $k$-starlike w.r.t. $w$ for $k=$ $2 \cos (\alpha \pi / 2)$. By Theorem 2.7 there exists $\varrho>0$ such that $\Omega(\xi, \varrho)$ is not $k$-starlike w.r.t. $w$. This means that there exists $w_{0} \in \Omega(\xi, \varrho)$ such that $E_{k}\left(w, w_{0}\right)$ is not contained in $\Omega(\xi, \varrho)$.

Suppose that

$$
\Gamma_{k}^{+}\left(w, w_{0}\right) \cap \gamma_{\varrho} \neq \emptyset
$$

Thus there exists $w_{1} \in\left(\Gamma_{k}^{+}\left(w, w_{0}\right) \backslash\left\{w, w_{0}\right\}\right) \cap \gamma_{\varrho}$ such that the subarc of $\Gamma_{k}^{+}\left(w, w_{0}\right)$ joining $w_{1}$ and $w_{0}$ without the endpoint $w_{1}$ is contained in $\Omega(\xi, \varrho)$. Since $w_{1} \in \gamma_{\varrho}$, there exists $z_{1} \in C(\varrho)$ such that $w_{1}=f\left(z_{1}\right)$. Let $\varphi\left(z_{1}\right)$ denote the directed angle defined by (3.9), where $z$ is replaced by $z_{1}$. The tangent line to the convex set $E_{k}\left(w, w_{0}\right)$ at $w_{1}$ is the boundary of two closed half-planes denoted by $H_{1}$ and $H_{2}$. One of them, say $H_{1}$, supports the set $E_{k}\left(w, w_{0}\right)$, the other $H_{2}$ contains it. Since $\gamma_{\varrho}$ is positively oriented, from the definition of $w_{1}$ it follows that the tangent vector $\tau\left(z_{1}\right)$ lies in $H_{2}$, and the vector $i\left(w_{1}-w\right)$ lies in $H_{1}$. Hence the angle $\varphi\left(z_{1}\right)$ is positive. Further, using Observation 2.1, the fact that $w_{1} \neq w_{0}$ and (3.9) we have

$$
\arg Q\left(z_{1}, \xi\right)=\varphi\left(z_{1}\right) \geq \theta\left(z_{1}\right)=\theta\left(\frac{w_{1}-w}{w_{0}-w}\right)>\theta(1)=\arccos \frac{k}{2}=\alpha \frac{\pi}{2},
$$

contrary to $(3.8)$. 
Suppose now that

$$
\Gamma_{k}^{-}\left(w, w_{0}\right) \cap \gamma_{\varrho} \neq \emptyset .
$$

Thus there exists $w_{2} \in\left(\Gamma_{k}^{-}\left(w, w_{0}\right) \backslash\left\{w, w_{0}\right\}\right) \cap \gamma_{\varrho}$ such that the subarc of $\Gamma_{k}^{-}\left(w, w_{0}\right)$ joining $w_{2}$ and $w_{0}$ without the endpoint $w_{2}$ is contained in $\Omega(\xi, \varrho)$. Let $z_{2} \in C(\varrho)$ be such that $w_{2}=f\left(z_{2}\right)$. Since $\gamma_{\varrho}$ is positively oriented, we see that the tangent vector $\tau\left(z_{2}\right)$ lies in the closed half-plane supporting $E_{k}\left(w, w_{0}\right)$ at $w_{2}$, and $i\left(f\left(z_{2}\right)-w\right)$ lies in the complementary closed half-plane. In consequence, the angle $\varphi\left(z_{2}\right)$ is negative. Moreover, by Observation 2.1, the fact that $w_{2} \neq w$ and (3.9) we have

$$
\arg Q\left(z_{2}, \xi\right)=\varphi\left(z_{2}\right) \leq \vartheta\left(z_{2}\right)=\vartheta\left(\frac{w_{2}-w}{w_{0}-w}\right)<\vartheta(1)=-\arccos \frac{k}{2}=-\alpha \frac{\pi}{2},
$$

which contradicts (3.8).

So $f \in S^{\mathrm{g}}(k ; \xi, w)$ with $k=2 \cos (\alpha \pi / 2)$, which ends the proof of the theorem.

4. Remarks. Taking into account (3.1) we can introduce the following

Definition 4.1. For each $\alpha \in(0,1]$ and $\xi \in U$ we denote by $S^{*}(\alpha ; \xi)$ the class of all functions $f$ regular in $U$ satisfying the condition

$$
\left|\arg \left\{\frac{(1-\bar{\xi} z)(z-\xi) f^{\prime}(z)}{f(z)-f(\xi)}\right\}\right|<\alpha \frac{\pi}{2}, \quad z \in U .
$$

From Theorem 3.1 it follows that every function in $S^{*}(\alpha ; \xi)$ is univalent and belongs to the unique class $S^{\mathrm{g}}(k ; \xi, w)$ for $k=2 \cos (\alpha \pi / 2)$.

Theorem 3.1 gives an equivalence between $k$-starlikeness with respect to a fixed point $w \in \mathbb{C}$, a property which defines the class $S^{\mathrm{g}}(k ; \xi, w)$, and an analytic condition (4.1) which describes the class $S^{*}(\alpha ; \xi)$, where $\alpha=(2 / \pi) \arccos (k / 2)$. For $\xi=0$ and $w=f(\xi)=0$ we get the results of Ma and Minda [3]. Then the inequality (4.1) reduces to (1.1) and with the normalization $f^{\prime}(0)=1$ defines the class $S^{*}(\alpha)$ of strongly starlike functions, which coincides with the subclass of $S^{\mathrm{g}}(k ; 0,0), k=2 \cos (\alpha \pi / 2)$, with standard normalization.

The subclass of $S^{*}(1 ; \xi)$ with normalization $f(0)=0$ is known. For details about this class see [2, Vol. I, pp. 155-164]. But this normalization seems to be unnatural. It excludes situations like $\xi=0$ and $w=f(\xi) \neq 0$ or $\xi \neq 0$ and $w=f(\xi)=0$.

It is also natural to consider the subclass of $S^{*}(\alpha ; \xi)$ with normalization $f^{\prime}(\xi)=1$. 
It is worth noticing that the condition (3.7) was obtained in 1978 by Wald [6], who transformed the condition

$$
\operatorname{Re}\left\{\frac{z f^{\prime}(z)}{f(z)-f(\xi)}\right\}>0, \quad z \in U,
$$

into the form (3.7). The inequality (4.2) says geometrically that the domains $f\left(U_{r}\right)$ are starlike with respect to $w=f(\xi)$ for all $r$ such that $|\xi|<r<1$. Since at $z=\xi$ the expression on the left side of (4.1) has a pole, this condition fails to characterize the class $\mathcal{Z}(w)$.

Looking at Theorem 2.7 it is clear that starlikeness of $f$ with respect to $w=f(\xi)$ is not connected with the family $U_{r}, r \in(0,1)$, of Euclidean disks but rather with the family of hyperbolic disks $B(\xi, \varrho)$ where $\varrho>0$. This last family is transformed by every function $f$ in $S^{*}(1 ; \xi)$ onto a family of starlike domains with respect to $f(\xi)$.

\section{References}

[1] D. A. Brannan and W. E. Kirwan, On some classes of bounded univalent functions, J. London Math. Soc. (2) 1 (1969), 431-443.

[2] A. W. Goodman, Univalent Functions, Mariner, Tampa, Fla., 1983.

[3] W. Ma and D. Minda, An internal geometric characterization of strongly starlike functions, Ann. Univ. Mariae Curie-Skłodowska Sect. A 45 (1991), 89-97.

[4] J. Stankiewicz, On a family of starlike functions, ibid. 22-24 (1968-70), 175-181.

[5] -, Quelques problèmes extrémaux dans les classes des fonctions $\alpha$-angulairement étoilées, ibid. 20 (1966), 59-75.

[6] J. K. Wald, On starlike functions, Ph.D. thesis, Univ. of Delaware, Newark, Del., 1978.

Department of Mathematics

Technical University of Rzeszów

W. Pola 2

35-959 Rzeszów, Poland

E-mail: alecko@prz.rzeszow.pl 